\title{
Protein expression profile changes of lung tissue in patients with pulmonary hypertension
}

\author{
Min Wu ${ }^{\text {Corresp., } 1}$, Yijin Wu ${ }^{1}$, Jinsong Huang ${ }^{1}$, Yueheng $\mathbf{W u}^{1}{ }^{1}$, Hongmei $\mathrm{Wu}^{1}{ }^{1}$, Benyuan Jiang ${ }^{1}$, Jian Zhuang ${ }^{\text {Corresp. } 1}$ \\ ${ }^{1}$ Department of Cardiac Surgery, Guangdong Provincial People's Hospital, Guangzhou City, Guangdong Province, China \\ Corresponding Authors: Min Wu, Jian Zhuang \\ Email address: wumin0011@gdph.org.cn, zhuangjian5413@tom.com
}

Background. Pulmonary hypertension occurs in approximately $1 \%$ of the global population, and the prognosis for such patients may be poor. However, the mechanisms underlying the development of this disease remain unclear. Thus, understanding the development of pulmonary hypertension and finding new therapeutic targets and approaches are important for improved clinical outcomes. Methods. Lung tissue specimens were collected from six patients with atrial septal defect and pulmonary hypertension (all women, with a mean age of $46.5 \pm 4.7$ years, and their condition could not be corrected with an internal medical occlusion device) and from nine control patients with lung cancer who underwent lobectomy (six men and three women, with a mean age of $56.7 \pm 1.7$ years). Isobaric tags for relative and absolute quantitation and liquid chromatography tandem mass spectrometry analyses were used to detect protein expression levels. Results. We found 74 significantly upregulated and 88 significantly downregulated differentially expressed proteins between control and pulmonary hypertensive lung tissue specimens. Gene ontology analyses identified the top 20 terms in all three categories, that is, biological process, cellular component, and molecular function. Kyoto Encyclopedia of Genes and Genomes and protein-protein interaction analyses determined the top 10 signaling pathways and found that the six hub proteins associated with the differentially expressed upregulated proteins (PRKAA1, DHPR, ACTB, desmin, ACTG1, and ITGA1) were all involved in hypertrophic cardiomyopathy, arrhythmogenic right ventricular cardiomyopathy, and dilated cardiomyopathy. Conclusion. Our results identified protein expression profile changes in lung tissue derived from patients with pulmonary hypertension, providing potential new biomarkers for clinical diagnosis and prognosis for patients with pulmonary hypertension and offering candidate protein targets for future therapeutic drug development. 
1 Protein expression profile changes of lung tissue in patients with pulmonary hypertension

3 Min Wu, Yijin Wu, Jinsong Huang, Yueheng Wu, Hongmei Wu, Benyuan Jiang, Jian Zhuang

4 Department of Cardiac Surgery, Guangdong Provincial People's Hospital, 96 Dongchuan Road,

5 Yuexiu Distinct, Guangzhou City, Guangdong Province 510080, China

6

7

8 Corresponding author:

9 Dr. Min $\mathrm{Wu}$

10 Department of Cardiac Surgery

11 Guangdong Provincial People’s Hospital

1296 Dongchuan Road, Yuexiu Distinct, Guangzhou City, Guangdong Province 510080, China

13 Tell: $+86-18925046785$

14 Email: wumin0011@gdph.org.cn

16 Professor Jian Zhuang

17 Department of Cardiac Surgery

18 Guangdong Provincial People’s Hospital

1996 Dongchuan Road, Yuexiu Distinct, Guangzhou City, Guangdong Province 510080, China

20 Tel: $+86-20-83827812$ 
21 Email: zhuangjian5413@tom.com

\section{Abstract}

24 Background. Pulmonary hypertension occurs in approximately $1 \%$ of the global population, and

25 the prognosis for such patients may be poor. However, the mechanisms underlying the

26 development of this disease remain unclear. Thus, understanding the development of pulmonary

27 hypertension and finding new therapeutic targets and approaches are important for improved

28 clinical outcomes.

29 Methods. Lung tissue specimens were collected from six patients with atrial septal defect and

30 pulmonary hypertension (all women, with a mean age of $46.5 \pm 4.7$ years, and their condition

31 could not be corrected with an internal medical occlusion device) and from nine control patients

32 with lung cancer who underwent lobectomy (six men and three women, with a mean age of 56.7

$33 \pm 1.7$ years). Isobaric tags for relative and absolute quantitation and liquid chromatography

34 tandem mass spectrometry analyses were used to detect protein expression levels.

35 Results. We found 74 significantly upregulated and 88 significantly downregulated differentially

36 expressed proteins between control and pulmonary hypertensive lung tissue specimens. Gene

37 ontology analyses identified the top 20 terms in all three categories, that is, biological process,

38 cellular component, and molecular function. Kyoto Encyclopedia of Genes and Genomes and

39 protein-protein interaction analyses determined the top 10 signaling pathways and found that the

40 six hub proteins associated with the differentially expressed upregulated proteins (PRKAA1, 
41 DHPR, ACTB, desmin, ACTG1, and ITGA1) were all involved in hypertrophic cardiomyopathy,

42 arrhythmogenic right ventricular cardiomyopathy, and dilated cardiomyopathy.

43 Conclusion. Our results identified protein expression profile changes in lung tissue derived from

44 patients with pulmonary hypertension, providing potential new biomarkers for clinical diagnosis

45 and prognosis for patients with pulmonary hypertension and offering candidate protein targets

46 for future therapeutic drug development.

47

48 Keywords: pulmonary hypertension, proteomics, LC-MS/MS, iTRAQ, PRKAA1, DHPR

Introduction

55 Pulmonary hypertension is a chronic, persistent complex disease involving many lesions (Kim \&

56 George 2019). The incidence of pulmonary hypertension is approximately $1 \%$ of the global

57 population (Hoeper et al. 2016). Epidemiological surveys in Europe and the United States show

58 that idiopathic pulmonary hypertension is most common in women and that familial pulmonary

59 hypertension is associated with genes (Franco et al. 2019; McGoon et al. 2013). Pulmonary

60 hypertension is a chromosomal dominant genetic disease mainly attributable to bone 
61 morphogenetic protein receptor-2 mutations (Harper et al. 2019). Other types of pulmonary

62 hypertension are often associated with a variety of diseases, including congenital heart disease,

63 chronic obstructive pulmonary disease, connective tissue disease, drug and toxin effects,

64 HIV/AIDS, hemoglobinopathy, and coagulation disorders (Basyal et al. 2019; Kim \& George

65 2019; Mitra et al. 2018; Zhang et al. 2019). Pulmonary hypertension can also be related to

66 certain atmospheric conditions, such as hypoxia observed at high altitude. Without effective

67 treatment, the prognosis for patients with pulmonary hypertensive is generally poor. The annual

68 mortality rate of such patients is approximately $15 \%$ (Thenappan et al. 2007). Poor

69 cardiopulmonary function, low mobility, increased right atrial pressure, progressive right

70 ventricular failure, low cardiac output, increased brain natriuretic peptide, and progression of

71 connective tissue disease are all predictors of poor prognosis (Fukuda et al. 2019).

73 Recent studies have revealed several new candidate targets and approaches for development of

74 future pulmonary hypertension treatment. Zhang et al. (2019) have suggested that autophagy of

75 pulmonary artery endothelial and smooth muscle cells may induce dysfunction of the pulmonary

76 arteries and eventually lead to pulmonary hypertension (Zhang et al. 2019). Lambert et al. (2018)

77 have reported that ion channel activity changes in pulmonary artery endothelial and smooth

78 muscle cells may pathophysiologically contribute to the development of pulmonary hypertension

79 (Lambert et al. 2018). Additionally, immune system dysfunction and inflammation may be

80 linked with pulmonary arterial hypertension pathogenesis (Goldenberg \& Steinberg 2019). 
81 Therefore, it is important to find new and effective targets to treat pulmonary hypertension.

82

83 Isobaric tags for relative and absolute quantitation (iTRAQ) in combination with liquid

84 chromatography tandem mass spectrometry (LC-MS/MS) are powerful tools for identifying

85 protein expression levels of the various types of proteins that are in a specimen. In the present

86 study, we obtained pulmonary tissue specimens from patients undergoing surgical pulmonary

87 procedures and used the iTRAQ and LC-MS/MS methods to identify proteins that were

88 differentially expressed in the pulmonary tissue of patients with pulmonary hypertension versus

89 those from patients without this disease. Using bioinformatics analysis, we assessed those

90 differentially expressed proteins (DEPs) to determine potential key proteins and their related

91 signaling pathways that may be associated with the development of pulmonary hypertension.

93 Materials \& Methods

94 Clinical specimens and patient enrollment

95 Our research plan and an informed patient consent form for the present study were submitted to

96 the Medical Research Ethics Committee of Guangdong Provincial People's Hospital. The

97 Medical Ethics Committee reviewed and approved our submitted proposal, ethical approval number Guangdong Medical Ethics 2016220H (R1)/GDREC2016220H (R1). All patients signed the written informed consent form prior to being included in the study. 
101 Patients were enrolled in strict accordance with the following inclusion and exclusion criteria, as

102 specified in the experimental protocol. For the experimental group, the included patients had

103 been diagnosed as having pulmonary hypertension with an atrial septal defect that could not be

104 corrected by internal closure. In the resting state, with the patient lying so that the left atrium was

105 in a horizontal position and the measurement determined at a distance midway between the

106 middle of the sternum and the bedside, the average pressure of the pulmonary artery measured by

107 a catheter located in the right ventricle of the heart was $\geq 25 \mathrm{mmHg}$. For the control group, the

108 included patients did not have pulmonary hypertension and had received a diagnosis of central

109 lung cancer requiring lobectomy, but the cancer tissue had not invaded the peripheral lung tissue

110 used in this study.

111

\section{2 iTRAQ labeling and fractionation by cation exchange chromatography}

113 The 8-plex iTRAQ labeling method was performed as previously described (Ding et al. 2019;

114 Liu et al. 2019). The reagents were used to label protein peptides from each group multiplex kit

115 (ABI, Foster City, CA, USA) (isobaric tags 113, 114, and 116 for the control group, and isobaric

116 tags 118,119 , and 121 for the pulmonary hypertension group). For each specimen, protein (200

$117 \mu \mathrm{g})$ was precipitated with acetone at $-20^{\circ} \mathrm{C}$ overnight. After centrifugation for $10 \mathrm{~min}$, the

118 protein pellet was dissolved in $60 \mu \mathrm{L}$ of iTRAQ dissolution buffer (Applied Biosystems). The

119 iTRAQ labeling reagents were added to the corresponding peptide specimen to react at room

120 temperature for $1 \mathrm{~h}$. After the reaction for the labeling was stopped and an extraction efficiency 
121 test was performed, the specimen was ZipTip desalted and subjected to a matrix-assisted laser

122 desorption ionization procedure. Each group was pooled and then vacuum-dried. Each pooled

123 specimen containing mixed peptides was lyophilized and dissolved in solution A (2\% acetonitrile

124 and $20 \mathrm{mM}$ ammonium formate, $\mathrm{pH}$ 10). This solution was loaded on a reverse-phase column

125 (Luna C18, $4.6 \times 150 \mathrm{~mm}$; Phenomenex; Torrance, CA, USA) and eluted using a step linear

126 elution program at a flow rate of $0.8 \mathrm{~mL} / \mathrm{min}$ : 0\%-10\% buffer B $\left(500 \mathrm{mM} \mathrm{KCl}, 10 \mathrm{mM} \mathrm{KH}_{2} \mathrm{PO}_{4}\right.$

127 in $25 \%$ acetonitrile, $\mathrm{pH} 2.7$ ) for $10 \mathrm{~min}, 10 \%-20 \%$ buffer $\mathrm{B}$ for $25 \mathrm{~min}, 20 \%-45 \%$ buffer B for 5

$128 \mathrm{~min}$, and $50 \%-100 \%$ buffer B for $5 \mathrm{~min}$. The eluent fractions were collected and centrifuged for

129 5-45 min. The fractions were finally combined into six pools and desalted using C18 Cartridges

130 (Empore standard density Solid Phase Extraction C18 Cartridges, bed I.D. 7 mm, 3 mL volume;

131 Sigma; St. Louis, MO, USA).

132

133 LC-MS/MS analysis

134 We performed MS using a TripleTOF 5600 system (AB SCIEX) combined with a nanoliter

135 spray III ion source (AB SCIEX, USA). The spray voltage was set at $2.5 \mathrm{kV}$; the air curtain

136 pressure, at $30 \mathrm{psi}$; the atomization pressure at $5 \mathrm{psi}$; and the heater temperature, at $150^{\circ} \mathrm{C}$. The

137 scanning mode used was information-dependent acquisition. The scan time of the first time-of-

138 flight (TOF)-MS single image was $250 \mathrm{~ms}$. Each information-dependent acquisition cycle

139 collected 35 secondary TOF-MS images. Each cycle time was fixed at $2.5 \mathrm{~s}$. The dynamic

140 exclusion was set to $18 \mathrm{~s}$, which was approximately equal to half the width of the chromatogram. 


\section{Sequence database search and data analysis}

143 The data were processed according to the flowchart shown in Figure 1. After the data were

144 collected, we used Protein Pilot Software v. 5.0 (AB SCIEX, USA) for the analysis. Human

145 proteome databases containing reviewed Uniprot sequences were used to perform peptide

146 identification. Decoys for the database search were generated with the revert function. We chose

147 the following options to identify the proteins: cysteine alkylation $=$ iodoacetamide; enzyme $=$

148 trypsin; search effort $=$ thorough. The proteins that showed a relative upregulated or

149 downregulated fold change of at least 1.2 (in both replicates) and that were significantly different

150 between the groups at $P<0.05$ were determined to be differentially expressed between the

151 groups.

152

153 Gene ontology (GO) and Kyoto Encyclopedia of Genes and Genomes (KEGG) pathway

154 enrichment analyses

155 We performed GO analyses using Metascape, a web-based resource for gene and protein

156 annotation, visualization, and integration discovery (http://metascape.org) (Fang et al. 2019;

157 Soonthornvacharin et al. 2017). KEGG pathway analyses of the DEPs were performed using the

158 KOBAS online analysis database (http://kobas.cbi.pku.edu.cn/) (Kanehisa \& Goto 2000). A two-

159 sided $P<0.05$ was considered statistically significant. 
161 Protein-protein interaction (PPI) network construction and module analyses

162 PPI analysis was used to assess the functions associated with the DEPs and to determine the

163 general organizational principles of the functional cellular networks. The functional relationships

164 between proteins were identified using the Search Tool for the Retrieval of Interacting Genes

165 (STRING; http://string.embl.de/) (Fang et al. 2019; von Mering et al. 2003). The PPI networks

166 associated with the respective DEPs were constructed to predict the interaction of those proteins.

168 Results

169 Specimen collection and patient characteristics

170 From April to September 2016, we collected lung tissue specimens from six patients with atrial

171 septal defect and pulmonary hypertension. The patients were all women, with a mean (SD) age

172 of $46.5 \pm 4.7$ years, and their condition could not be corrected with an internal medical occlusion

173 device. For the control group, nine patients with lung cancer who underwent lobectomy were

174 enrolled: six men and three women, with a mean (SD) age of $56.7 \pm 1.7$ years. Among the

175 patients with pulmonary hypertension, the mean pulmonary systolic pressure was $66.7 \pm 5.5$

$176 \mathrm{mmHg}$; mean pulmonary artery diastolic pressure, $23.2 \pm 1.6 \mathrm{mmHg}$; mean pulmonary artery

177 pressure, $39.7 \pm 3.4 \mathrm{mmHg}$; mean pulmonary artery resistance, $3.27 \pm 0.28$ Wood units;

178 pulmonary oxygen saturation, $87.3 \% \pm 0.84 \%$; and mean patient body surface, $1.52 \pm 0.06 \mathrm{~m}^{2}$.

179

180

Identification of DEPs in pulmonary tissue 
181 After performing the quantitative analyses with Protein Pilot Software, we identified 2953

182 proteins using a detection protein threshold (Unused ProtScore [Conf]) cutoff of $>1.3 ; 95 \%$

183 confidential interval) (Table 1), and we obtained the protein expression fold changes between the

184 control specimens and the pulmonary hypertensive lung tissue specimens. The data indicated

185 marked protein expression profile changes between the control lung tissue and the pulmonary

186 hypertensive lung tissue. Using our selection criteria, we identified 74 significantly upregulated

187 DEPs, including integrin subunit $\alpha 1$ (ITGA1) and the voltage-gated calcium $\left(\mathrm{Ca}^{2+}\right)$ channel

$188 \alpha 2 / \delta 1$ subunit, and 88 significantly downregulated DEPs, including Rho-GDP dissociation

189 inhibitor 2 (Table 2, Figure 2).

190

191 GO functional enrichment analysis

192 GO analysis is used to hierarchically classify genes or gene products into categories organized in

193 an ontology. The analysis is based on three categories: (1) molecular function, to describe the

194 molecular activity of a gene; (2) biological process, to characterize the larger cellular or

195 physiological role of the gene; and (3) cellular component, to indicate the location of the gene or

196 gene product in the cell. We used Metascape to enrich all identified proteins. Among the top 20

197 terms in the biological process category, the highest percentages of the proteins were associated

198 with the term single-organism process (Figures 3 and 4$)(n=119$ proteins; with the top 3

199 upregulated proteins in this process being LDLRAP1, FTL, and CHUK). The maximum levels of

200 the expressed proteins this category were detected for the term protein complex subunit 
201 organization $(\mathrm{n}=27$ proteins; with the top 3 upregulated proteins being SLC6A4, FRYL, and

202 HIST2H3A). Among the top 20 terms in the GO category cellular component, the highest

203 percentages of proteins were associated with the term cytoplasm $(n=104$ proteins, with the top 3

204 upregulated proteins for this term being LDLRAP1, FTL and CHUK. The maximum levels of

205 the expressed proteins in this category were in the term major histocompatibility complex

206 (MHC), which is a group of proteins located on the cell surface ( $n=9$ proteins, with the top 2

207 upregulated proteins being HLA-A and HLA-DPA1). In the top 20 terms of the Go category

208 molecular function, the highest percentages of proteins were associated with the term binding

209 (113 proteins, with the top 3 upregulated proteins being LDLRAP1, FTL and CHUK), and the

210 maximum levels of the expressed proteins in this category were for the term ferric iron binding

211 (with the top 2 upregulated proteins being FTL and FTH1). The GO functional enrichment

212 analysis assessing the distributions of the proteins with the greatest fold changes in the biological

213 process, molecular function, and cellular component categories indicated that the proteins

214 associated with the terms organism process, protein binding, and extracellular vesicle,

215 respectively, were markedly changed.

216

217 KEGG signaling pathway analysis and PPI network construction

218 To identify the functions associated with the DEPs, we used the KOBAS online analysis tool to

219 determine the KEGG signaling pathways, and we used the STRING online analysis tool to

220 construct the PPI networks. Through KEGG signaling pathway analysis, the gene content in the 
221 provided genome is compared with the information in the KEGG pathway database to clarify

222 which pathways and associated functions link to the genes in the genome. The PPI network

223 indicates physical contacts between two or more proteins resulting from biochemical events

224 controlled by electrostatic forces, including hydrophobic effects. The top 10 KEGG signaling

225 pathways were enriched, and the PPI networks were constructed (Figures 5-9). We found that

226 the top 10 signaling pathways included hypertrophic cardiomyopathy, systemic lupus

227 erythematosus, arrhythmogenic right ventricular cardiomyopathy, dilated cardiomyopathy,

228 pathogenic Escherichia coli infection, viral myocarditis, phagosome, alcoholism, cardiac muscle

229 contraction, and ribosomes, which are predominantly associated with human diseases (Figure 5).

230 Some of the DEPs crossed several different signaling pathways. For example, the proteins

231 highlighted in red boxes in Figures 6-8, including DHPR, ACTB, desmin, ACTG1, PRKAA1

232 and ITGA1, were all involved in hypertrophic cardiomyopathy, arrhythmogenic right ventricular

233 cardiomyopathy, and dilated cardiomyopathy (Table 2). The results of the KEGG pathway

234 enrichment and PPI network construction analyses indicated that many of the upregulated

235 proteins (shown as brown dots in Figure 9) were involved in hypertrophic cardiomyopathy, By

236 contrast, many of the downregulated proteins (shown as green dots in Figure 9) were related to

237 ribosome function. Taken together, our results suggest that the heart is a major organ impaired

238 by pulmonary hypertension. 
241 In the present study, we used clinical specimens obtained from patients undergoing surgical

242 procedures, and we used the iTRAQ method combined with LC-MS/MS to identify the key

243 proteins and signaling pathways associated with the development of pulmonary hypertension.

244 Our primary findings were as follows: (1) We identified 2953 proteins, including 74 significantly

245 upregulated DEPs and 88 significantly downregulated DEPs, between control and pulmonary

246 hypertensive lung tissues; (2) GO analysis elucidated the top 20 terms associated with categories

247 of biological process, cellular component, and molecular function; (3) KEGG and PPI analyses

248 identified the top 10 signaling pathways and six hub proteins, including PRKAA1, DHPR,

249 ACTB, desmin, ACTG1 and ITGA1. Taken together, our study revealed protein expression

250 profile changes in lung tissue of patients with pulmonary hypertension, providing a deeper

251 understanding of the development of pulmonary hypertension and suggesting several potential

252 targets for the development of new drugs in the treatment of pulmonary hypertension.

254 In the present study, we detected a high expression of PRKAA1, which encodes 5'-AMP-

255 activated protein kinase catalytic subunit alpha-1 (AMPK $\alpha 1$ ). AMPK $\alpha 1$ is an enzyme involved

256 in the pathological changes of smooth muscle cells in vessels of patients with pulmonary

257 hypertension. AMPK $\alpha 1$ can maintain smooth muscle cells and their survival in an anoxic

258 environment, and inhibition of AMPK $\alpha 1$ leads to hypoxia-induced activation of autophagy. In a

259 hypoxic environment, AMPK is activated and phosphorylated in smooth muscle cells. Under

260 hypoxic conditions, lactate dehydrogenase activity is increased after AMPK activity is inhibited 
261 by the AMPK antagonist compound C, leading to the induction of apoptosis in smooth muscle

262 cells (Ibe et al. 2013). Evans et al. (2015) have suggested that the levels of AMPK and liver

263 kinase B1 (LKB1) are upregulated when mitochondria sense a change in the oxygen supply.

264 These upregulated levels cause a sustained blood vessel contraction through an LKB1-AMPK

265 signaling pathway and result in continuous pulmonary hypertension. AMPK is activated by an

266 increase in the ratio of ADP to ATP and rapidly amplifies the signaling pathway in synergy with

267 LKB1, maintaining the intracellular ATP concentration by catabolism and inhibition of

268 unnecessary ATP depletion. Therefore, AMPK regulates the mitochondrial energy balance and

269 maintains the energy supply of smooth muscle cells during hypoxia to preserve normal

270 physiological functions (Evans et al. 2015). In the present study, the expression of AMPK $\alpha 1$ was

271 significantly increased in the lung tissue of patients with pulmonary hypertension. This finding

272 suggests that AMPK may be a potential target in the treatment of pulmonary hypertension.

273

274 We also found an upregulation of ITGA1, which is involved in the regulation of phosphorylation

275 and the biological processes of essential metabolic processes. The integrins $\alpha 1-5, \alpha 7-8, \alpha \mathrm{v}, \beta 1$,

$276 \beta 3$, and $\beta 4$ have been detected on pulmonary vessels. Umesh et al. used a mouse model of

277 chronic hypoxic pulmonary hypertension and performed immunohistochemical staining of

278 pulmonary artery smooth cells to find that the level of ITGA1 expression on the cell surface was

279 significantly increased, whereas that of integrin subunit $\alpha 5$ was significantly decreased (Umesh

280 et al. 2011; Umesh et al. 2006). These expression level changes were mainly concentrated on the 
281 smooth muscle cells of peripheral pulmonary arterioles and were associated with $\mathrm{Ca}^{2+}$ flow in

282 smooth muscle cells (Umesh et al. 2011). Eventually, a $\mathrm{Ca}^{2+}$ concentration change in vascular

283 smooth muscle cells affects blood vessel contraction and blood pressure. In the present study, we

284 also found an increase in ITGA1 in the lung tissue of patients with pulmonary hypertension.

285 Thus, the increased level of this integrin may be a key reason for the development of pulmonary

286 hypertension.

287

288 Smooth muscle cell contraction is regulated by intracellular $\mathrm{Ca}^{2+}$ and $\mathrm{Rho}$ kinase signaling

289 pathways (Berridge 2008). $\mathrm{Ca}^{2+}$ channels are involved in the pathological processes associated

290 with pulmonary hypertension. Thus, $\mathrm{Ca}^{2+}$ channel antagonists have been used in the early stages

291 of clinical treatment of pulmonary hypertension, and some patients have achieved some

292 therapeutic effects (Kennedy et al. 1985; Packer 1985; Packer et al. 1984; Rubin 1985). In the

293 present study, we found that the voltage-gated $\mathrm{Ca}^{2+}$ channel subunit $\alpha 2 / \delta 1$ encoded by $C A C N 2 D 1$

294 significantly increased in the lung tissue of patients with pulmonary hypertension. Moreover, we

295 also found that the $A R H G D I B$-encoded Rho-GDP dissociation inhibitor 2 protein is

296 downregulated (Table 2). Rho-GDP dissociation inhibitor 2 can inhibit the dissociation of GDP

297 from Rho protein, thereby regulating the rate of the GDP/GTP exchange reaction and inhibiting

298 smooth muscle cell contraction (Adra et al. 1993; Scherle et al. 1993). Therefore, an increase in

299 voltage-gated $\mathrm{Ca}^{2+}$ channels and a decrease in Rho-GDP dissociation inhibitor 2 levels will

300 eventually induce smooth muscle cell contraction, increase vascular resistance, and participate in 
301 the pathogenesis of pulmonary hypertension.

302

303 In the present study, we also found that three muscle contraction-related proteins, including $\beta$ -

304 actin encoded by $A C T B, \gamma$-actin encoded by $A C T G 1$, and desmin encoded by $D E S$, were

305 significantly increased. Beta-actin is one of two nonmuscle cytoskeletal actins, and it is involved

306 in cell motility, structure, and integrity (Gunning et al. 2015). Alpha-actins are a major

307 constituent of the contractile apparatus (Yonemura 2017). In adult striated muscle cells, $\gamma$-actin

308 localizes to Z-disks and costamere structures and is involved in force transduction and

309 transmission in muscle cells (Nakata et al. 2001; Papponen et al. 2009). Desmin is a type III

310 intermediate filament in cardiac muscle, skeletal muscle, and smooth muscle tissue. It integrates

311 the sarcolemma, $\mathrm{Z}$ disk, and nuclear membrane in sarcomeres and regulates sarcomere

312 architecture (Brodehl et al. 2018; Sequeira et al. 2014). Therefore, these upregulated muscle

313 contraction-related proteins may enhance vascular smooth muscle cell contraction to increase

314 vascular resistance and may thus be involved in the pathogenesis of pulmonary hypertension.

315

\section{Conclusions}

317 We used the iTRAQ method to identify 2953 proteins, including 74 significantly upregulated

318 DEPs and 88 significantly downregulated DEPs between control and pulmonary hypertensive

319 lung tissues. Through bioinformatics analyses, we found six increased key proteins, including

320 PRKAA1, DHPR, ACTB, desmin, ACTG1, and ITGA1. The findings of the present study 
321 provide potential new biomarkers for clinical diagnosis and prognosis for pulmonary

322 hypertension and candidate protein targets for drug development.

323

324 References:

325 Adra CN, Ko J, Leonard D, Wirth LJ, Cerione RA, and Lim B. 1993. Identification of a novel 326 protein with GDP dissociation inhibitor activity for the ras-like proteins CDC42Hs and rac I. Genes Chromosomes Cancer 8:253-261.

Basyal B, Jarrett H, and Barnett CF. 2019. Pulmonary Hypertension in HIV. Can J Cardiol $35: 288-298$.

Berridge MJ. 2008. Smooth muscle cell calcium activation mechanisms. J Physiol 586:50475061.

Brodehl A, Gaertner-Rommel A, and Milting H. 2018. Molecular insights into cardiomyopathies associated with desmin (DES) mutations. Biophys Rev 10:983-1006.

Ding F, Sun K, Sun N, Jiang Q, Cao M, and Wu Z. 2019. iTRAQ-based proteomics reveals SOD2 as a potential salivary biomarker in liver cancer. Int J Biol Markers:1724600819841619.

Evans AM, Lewis SA, Ogunbayo OA, and Moral-Sanz J. 2015. Modulation of the LKB1-AMPK Hypertension. Adv Exp Med Biol 860:89-99. 
341 Aberrantly hydroxymethylated differentially expressed genes and the associated protein 342 pathways in osteoarthritis. PeerJ 7:e6425.

343 Franco V, Ryan JJ, and McLaughlin VV. 2019. Pulmonary Hypertension in Women. Heart Fail Clin 15:137-145.

345 Fukuda K, Date H, Doi S, Fukumoto Y, Fukushima N, Hatano M, Ito H, Kuwana M, Matsubara H, Momomura SI, Nishimura M, Ogino H, Satoh T, Shimokawa H, Yamauchi-Takihara

Goldenberg NM, and Steinberg BE. 2019. Inflammation Drives Pulmonary Arterial Treatment of Pulmonary Hypertension (JCS 2017/JPCPHS 2017). Circ J 83:842-945.

355 Gunning PW, Ghoshdastider U, Whitaker S, Popp D, and Robinson RC. 2015. The evolution of compositionally and functionally distinct actin filaments. J Cell Sci 128:2009-2019.

Harper RL, Maiolo S, Ward RJ, Seyfang J, Cockshell MP, Bonder CS, and Reynolds PN. 2019. BMPR2-expressing bone marrow-derived endothelial-like progenitor cells alleviate pulmonary arterial hypertension in vivo. Respirology.

360 Hoeper MM, Humbert M, Souza R, Idrees M, Kawut SM, Sliwa-Hahnle K, Jing ZC, and Gibbs 
JS. 2016. A global view of pulmonary hypertension. Lancet Respir Med 4:306-322.

362 Ibe JC, Zhou Q, Chen T, Tang H, Yuan JX, Raj JU, and Zhou G. 2013. Adenosine

363

364

365

366

367

368

369

370

371

372

373

374

375

376

377

378

379

380 monophosphate-activated protein kinase is required for pulmonary artery smooth muscle cell survival and the development of hypoxic pulmonary hypertension. Am J Respir Cell Mol Biol 49:609-618.

Kanehisa M, and Goto S. 2000. KEGG: kyoto encyclopedia of genes and genomes. Nucleic Acids Res 28:27-30.

Kennedy TP, Michael JR, and Summer W. 1985. Calcium channel blockers in hypoxic pulmonary hypertension. Am J Med 78:18-26.

Kim D, and George MP. 2019. Pulmonary Hypertension. Med Clin North Am 103:413-423.

Lambert M, Capuano V, Olschewski A, Sabourin J, Nagaraj C, Girerd B, Weatherald J, Humbert M, and Antigny F. 2018. Ion Channels in Pulmonary Hypertension: A Therapeutic Interest? Int J Mol Sci 19.

Liu F, Liu XX, Liu X, Li T, Zhu P, Liu Z, Xue H, Wang W, Jr., Yang X, Liu J, and Han W. 2019. Integrated analyses of phenotype and quantitative proteome of CMTM4 deficient mice reveal its association with male fertility. Mol Cell Proteomics.

McGoon MD, Benza RL, Escribano-Subias P, Jiang X, Miller DP, Peacock AJ, Pepke-Zaba J, Pulido T, Rich S, Rosenkranz S, Suissa S, and Humbert M. 2013. Pulmonary arterial hypertension: epidemiology and registries. J Am Coll Cardiol 62:D51-59.

Mitra A, Ghosh RK, Bandyopadhyay D, Ghosh GC, Kalra A, and Lavie CJ. 2018. Significance 
of Pulmonary Hypertension in Hypertrophic Cardiomyopathy. Curr Probl Cardiol.

382 Nakata T, Nishina Y, and Yorifuji H. 2001. Cytoplasmic gamma actin as a Z-disc protein. Biochem Biophys Res Commun 286:156-163.

384

386

Packer M. 1985. Therapeutic application of calcium-channel antagonists for pulmonary hypertension. Am J Cardiol 55:196B-201B.

Packer M, Medina N, and Yushak M. 1984. Adverse hemodynamic and clinical effects of calcium channel blockade in pulmonary hypertension secondary to obliterative pulmonary vascular disease. J Am Coll Cardiol 4:890-901.

Papponen H, Kaisto T, Leinonen S, Kaakinen M, and Metsikko K. 2009. Evidence for gammaactin as a $\mathrm{Z}$ disc component in skeletal myofibers. Exp Cell Res 315:218-225.

Rubin LJ. 1985. Calcium channel blockers in primary pulmonary hypertension. Chest 88:257S260S.

Scherle P, Behrens T, and Staudt LM. 1993. Ly-GDI, a GDP-dissociation inhibitor of the RhoA GTP-binding protein, is expressed preferentially in lymphocytes. Proc Natl Acad Sci US A 90:7568-7572.

Sequeira V, Nijenkamp LL, Regan JA, and van der Velden J. 2014. The physiological role of cardiac cytoskeleton and its alterations in heart failure. Biochim Biophys Acta 1838:700722.

Soonthornvacharin S, Rodriguez-Frandsen A, Zhou Y, Galvez F, Huffmaster NJ, Tripathi S, Balasubramaniam VR, Inoue A, de Castro E, Moulton H, Stein DA, Sanchez-Aparicio 
401 MT, De Jesus PD, Nguyen Q, Konig R, Krogan NJ, Garcia-Sastre A, Yoh SM, and

402 Chanda SK. 2017. Systems-based analysis of RIG-I-dependent signalling identifies KHSRP as an inhibitor of RIG-I receptor activation. Nat Microbiol 2:17022.

404 Thenappan T, Shah SJ, Rich S, and Gomberg-Maitland M. 2007. A USA-based registry for 405 pulmonary arterial hypertension: 1982-2006. Eur Respir J 30:1103-1110.

406 Umesh A, Paudel O, Cao YN, Myers AC, and Sham JS. 2011. Alteration of pulmonary artery integrin levels in chronic hypoxia and monocrotaline-induced pulmonary hypertension. $J$ Vasc Res 48:525-537.

Umesh A, Thompson MA, Chini EN, Yip KP, and Sham JS. 2006. Integrin ligands mobilize $\mathrm{Ca} 2+$ from ryanodine receptor-gated stores and lysosome-related acidic organelles in pulmonary arterial smooth muscle cells. J Biol Chem 281:34312-34323. of predicted functional associations between proteins. Nucleic Acids Res 31:258-261.

414 Yonemura S. 2017. Actin filament association at adherens junctions. J Med Invest 64:14-19.

415 Zhang CF, Zhao FY, Xu SL, Liu J, Xing XQ, and Yang J. 2019. Autophagy in pulmonary 416 hypertension: Emerging roles and therapeutic implications. J Cell Physiol. 
Figure Legends

421 Figure 1. Flowchart of the data analysis procedure.

422

423 Figure 2. Differential protein expression. (A) Volcano plot with red dots on the right-hand side

424 indicating upregulation, red points on the left-hand side indicating downregulation, and black

425 dots indicating no significant change in protein expression levels based on the criteria of an

426 absolute $\log 2$ fold change $(\mathrm{FC})>1.2$ and $P<0.05$. (B) Bar graph indicating 74 upregulated and

42788 downregulated proteins. DEP indicates differentially expressed protein.

428

429

Figure 3. Enrichment of the gene ontology functional terms in the top 20 entries of the

three categories of biological process, cellular component, and molecular function for

highly expressed proteins. The entries in each category are sorted from left to right according to

their $-\log P$ value. The more significant, and the number of proteins corresponding to each item,

processing and presentation of ..." is antigen processing and presentation of peptide or polysaccharide antigen via MHC class II.

Figure 4. Maximum level corresponding to the top 20 proteins in the gene ontology 
440 function (C), and the percentages of enriched proteins. The ordinate is arranged from low to

441 high according to the maximum level, and each level is arranged by $P$ value. The abscissa is the

442 percentages of the enriched proteins. The number at the end of each bar is the number of proteins

443 enriched in the biological process, cellular component, or molecular function gene ontology

444 category. In A, the full name of "antigen processing and presentation of ..." is antigen

445 processing and presentation of peptide or polysaccharide antigen via MHC class II.

446

447 Figure 5. Kyoto Encyclopedia of Genes and Genomes (KEGG) signaling pathways. Top 10

448 KEGG signaling pathways (A) and KEGG classification histogram (B). In B, all enriched

449 pathways with significant differences in $P$ values are shown. The ordinate is the specific pathway

450 classification and name. On the right, the number of associated genes, $P$ values, and their

451 corresponding KEGG classifications are shown. The abscissa gives the percentages of the genes.

452

453

Figure 6. Changes in the hypertrophic cardiomyopathy signaling pathway in lung tissue of

patients with pulmonary hypertension. Upregulated proteins are highlighted in red boxes.

Figure 7. Changes in the arrhythmogenic right ventricular cardiomyopathy signaling

pathway in lung tissue of patients with pulmonary hypertension. Upregulated proteins are

highlighted in red boxes. 
460 Figure 8. Changes in the viral myocarditis signaling pathway in lung tissue of patients with

461 pulmonary hypertension. Upregulated proteins are highlighted in red boxes.

462

463 Figure 9. Protein-protein interaction networks in the Kyoto Encyclopedia of Genes and

464 Genomes pathways that are ranked as the top 10 regulatory pathways. 
Figure 1

Flowchart of the data analysis procedure.

\section{Raw data}

Protein pilot

\section{Sequence database search}

\section{Differentially expressed proteins}

Functional analysis

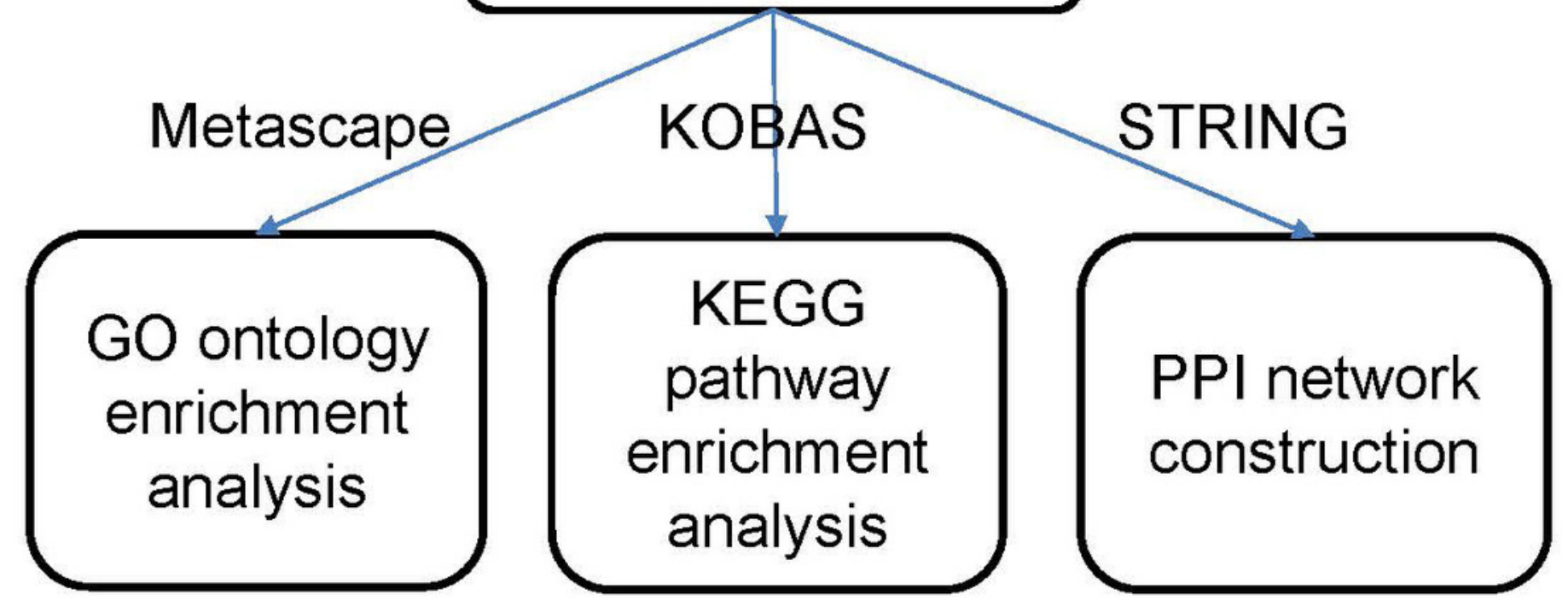




\section{Figure 2}

Differential protein expression.

(A) Volcano plot with red dots on the right-hand side indicating upregulation, red points on the left-hand side indicating downregulation, and black dots indicating no significant change in protein expression levels based on the criteria of an absolute log2 fold change $(F C)>1.2$ and $P<0.05$. (B) Bar graph indicating 74 upregulated and 88 downregulated proteins. DEP indicates differentially expressed protein.
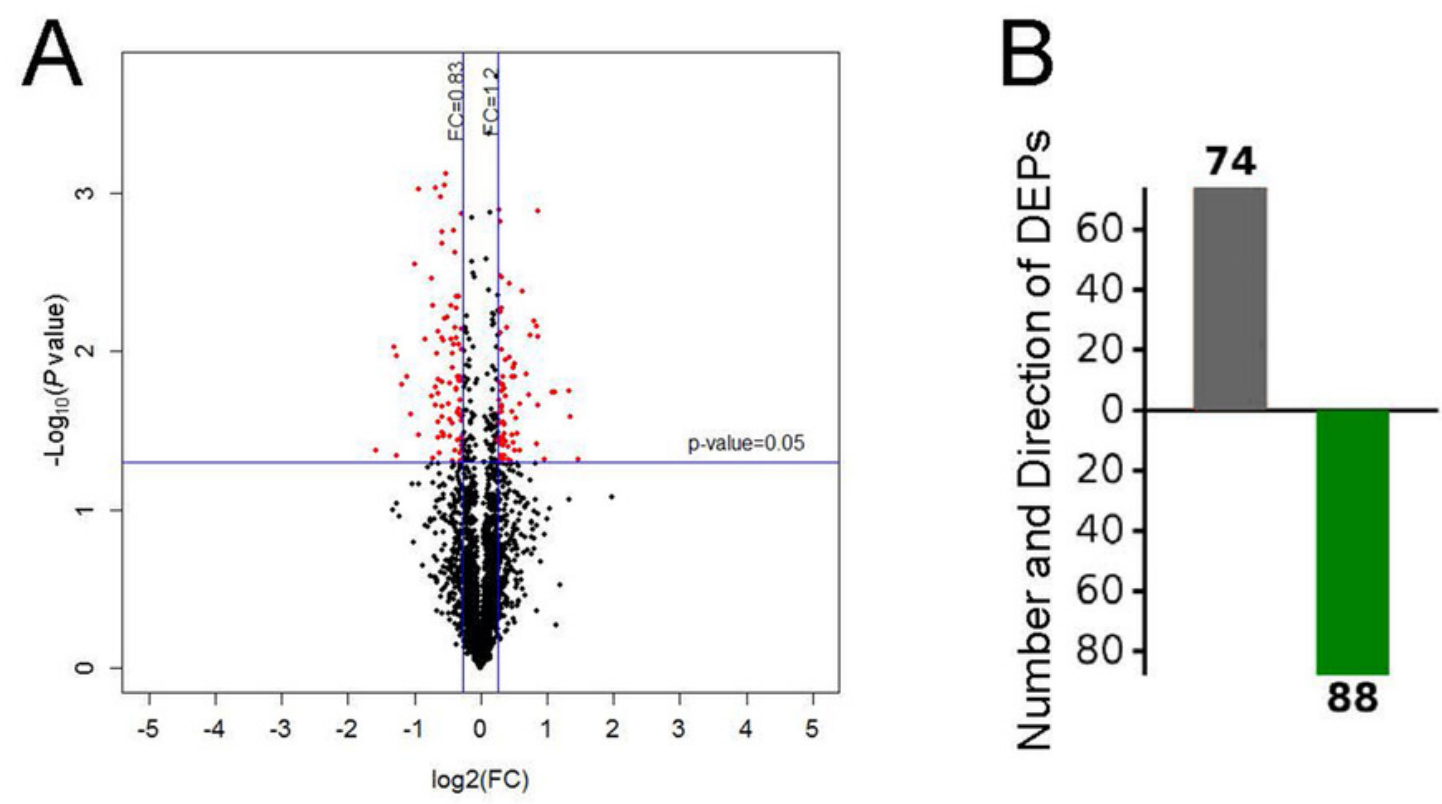

Upregulated Downregulated 


\section{Figure 3}

Enrichment of the gene ontology functional terms in the top 20 entries of the three categories of biological process, cellular component, and molecular function for highly expressed proteins.

The entries in each category are sorted from left to right according to their $-\log P$ value. The more significant, and the number of proteins corresponding to each item, can be viewed from the left to right vertical axes. The number of proteins is a percentage of the total number of submitted proteins. For the category biological process, the full name of "antigen processing and presentation of ..." is antigen processing and presentation of peptide or polysaccharide antigen via MHC class II.

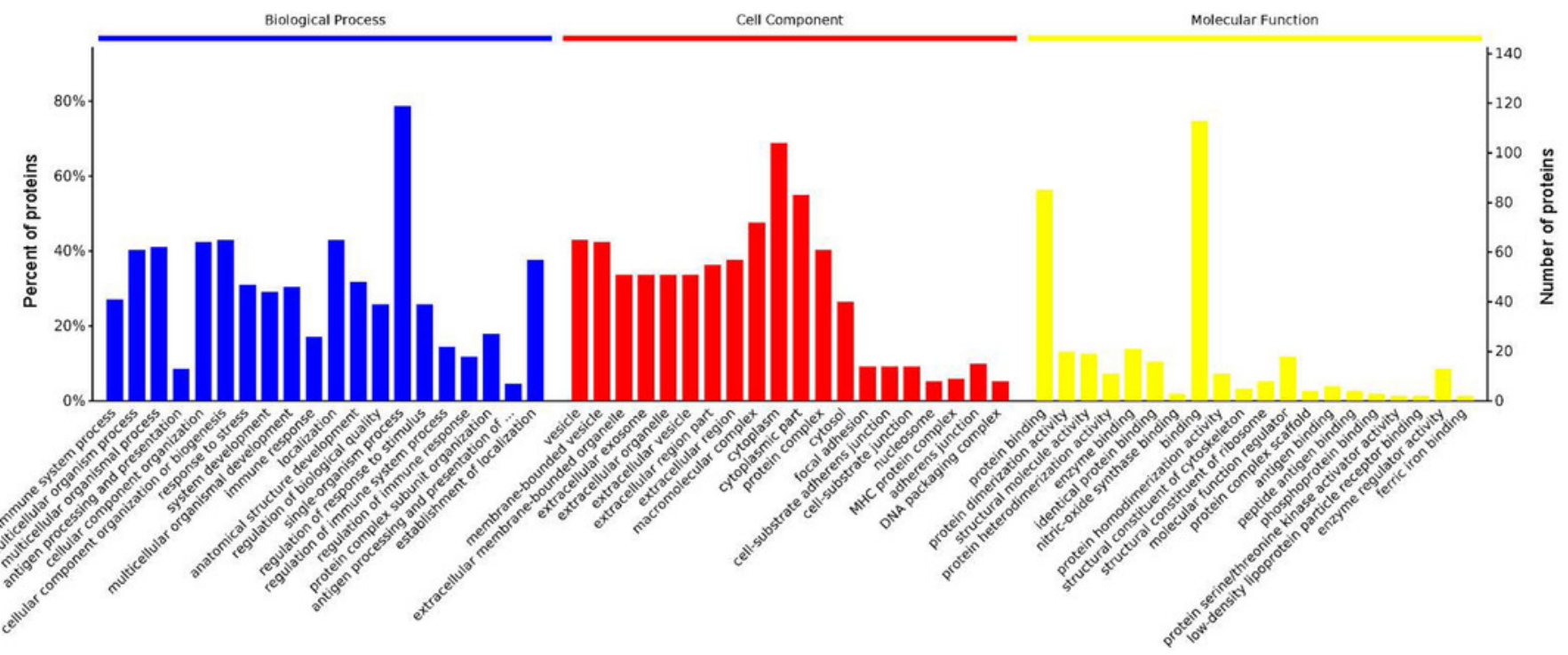




\section{Figure 4}

Maximum level corresponding to the top 20 proteins in the gene ontology enrichment categories of biological process (A), cellular component (B), and molecular function (C), and the percentages of enriched proteins.

The ordinate is arranged from low to high according to the maximum level, and each level is arranged by $P$ value. The abscissa is the percentages of the enriched proteins. The number at the end of each bar is the number of proteins enriched in the biological process, cellular component, or molecular function gene ontology category. In A, the full name of "antigen processing and presentation of ..." is antigen processing and presentation of peptide or polysaccharide antigen via MHC class II. 


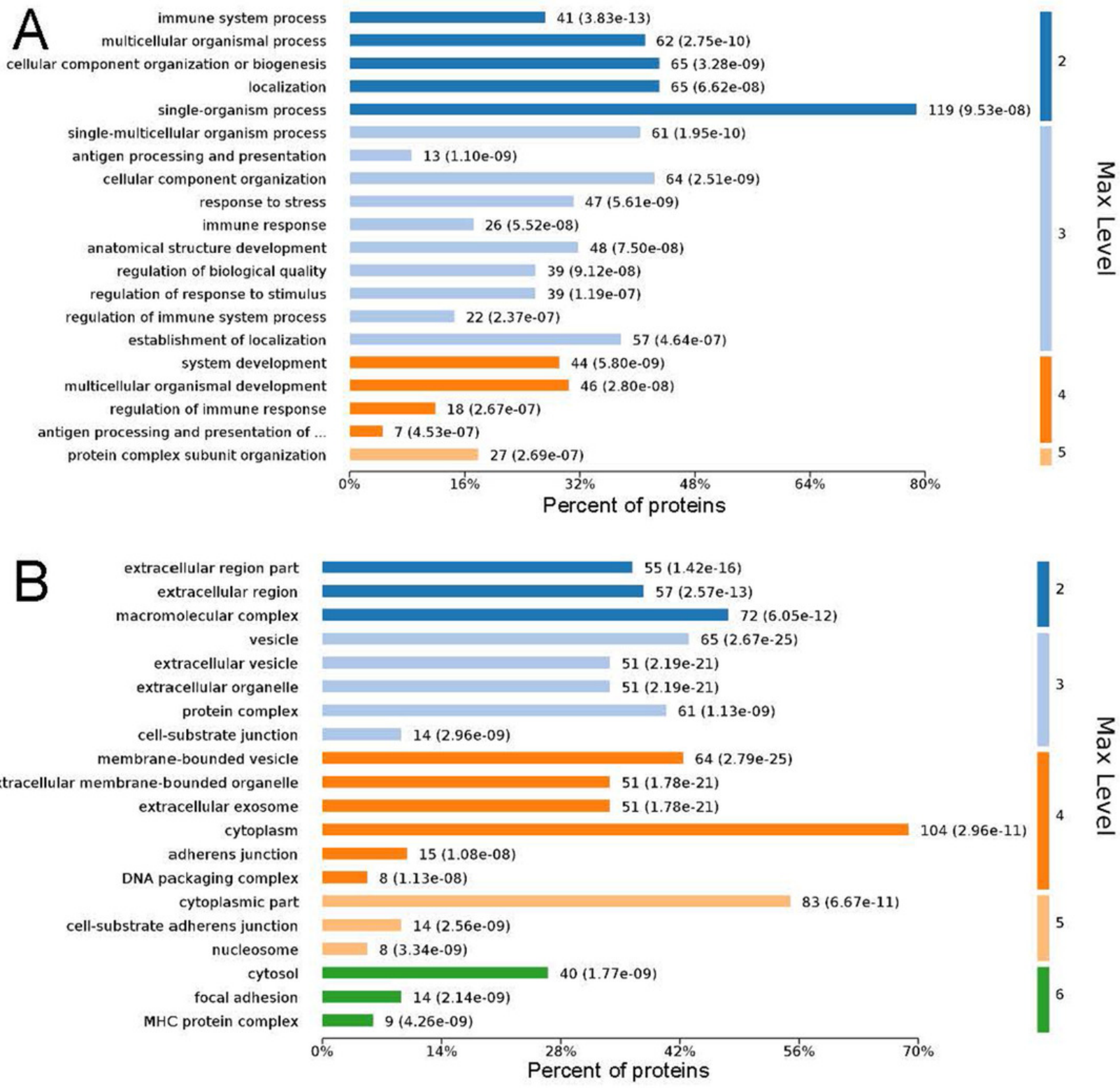

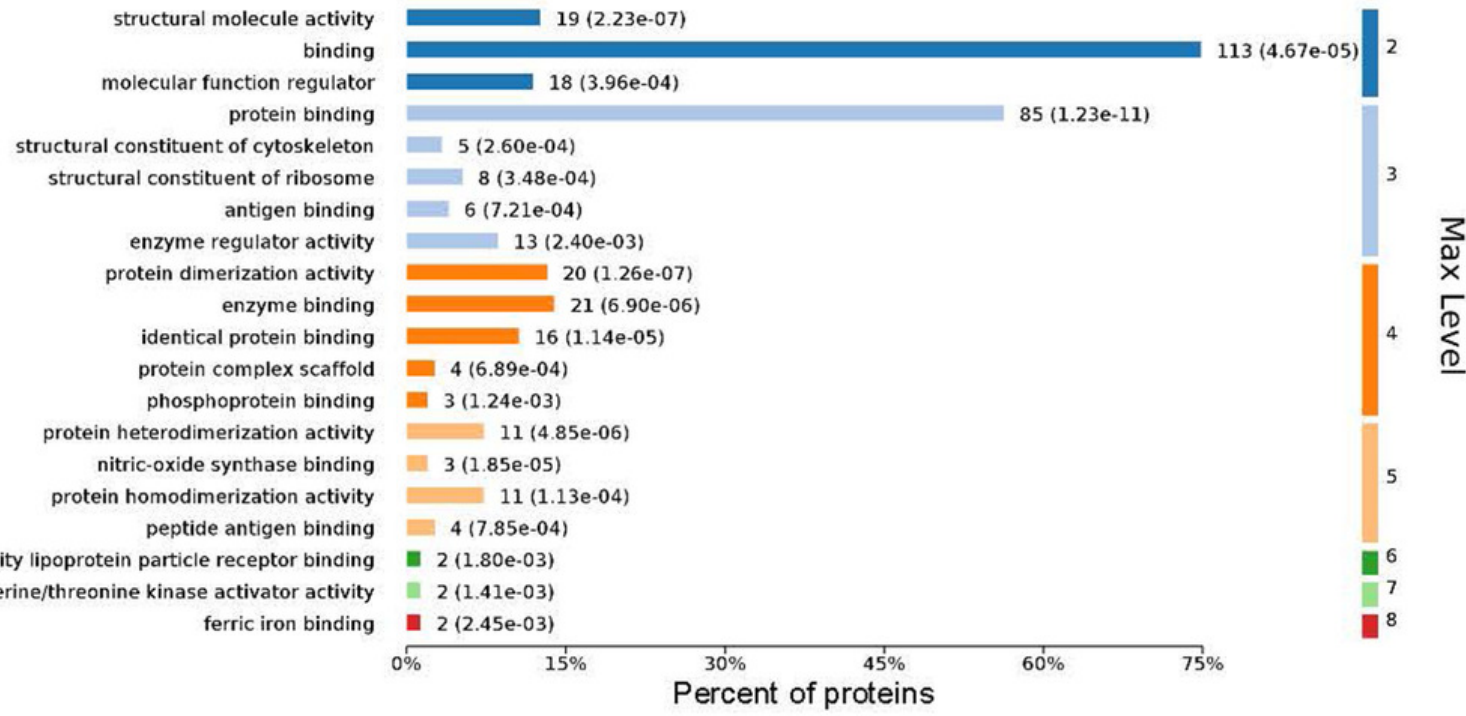




\section{Figure 5}

Kyoto Encyclopedia of Genes and Genomes (KEGG) signaling pathways.

Top 10 KEGG signaling pathways (A) and KEGG classification histogram (B). In B, all enriched pathways with significant differences in $\mathrm{P}$ values are shown. The ordinate is the specific pathway classification and name. On the right, the number of associated genes, $\mathrm{P}$ values, and their corresponding KEGG classifications are shown. The abscissa gives the percentages of the genes. 


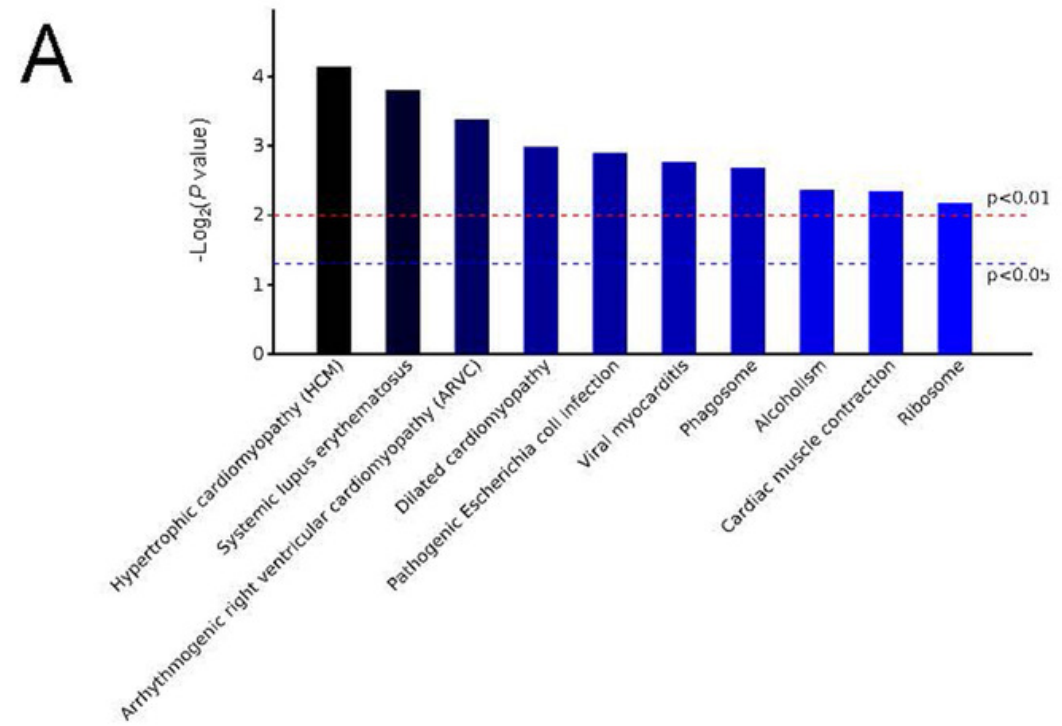

B

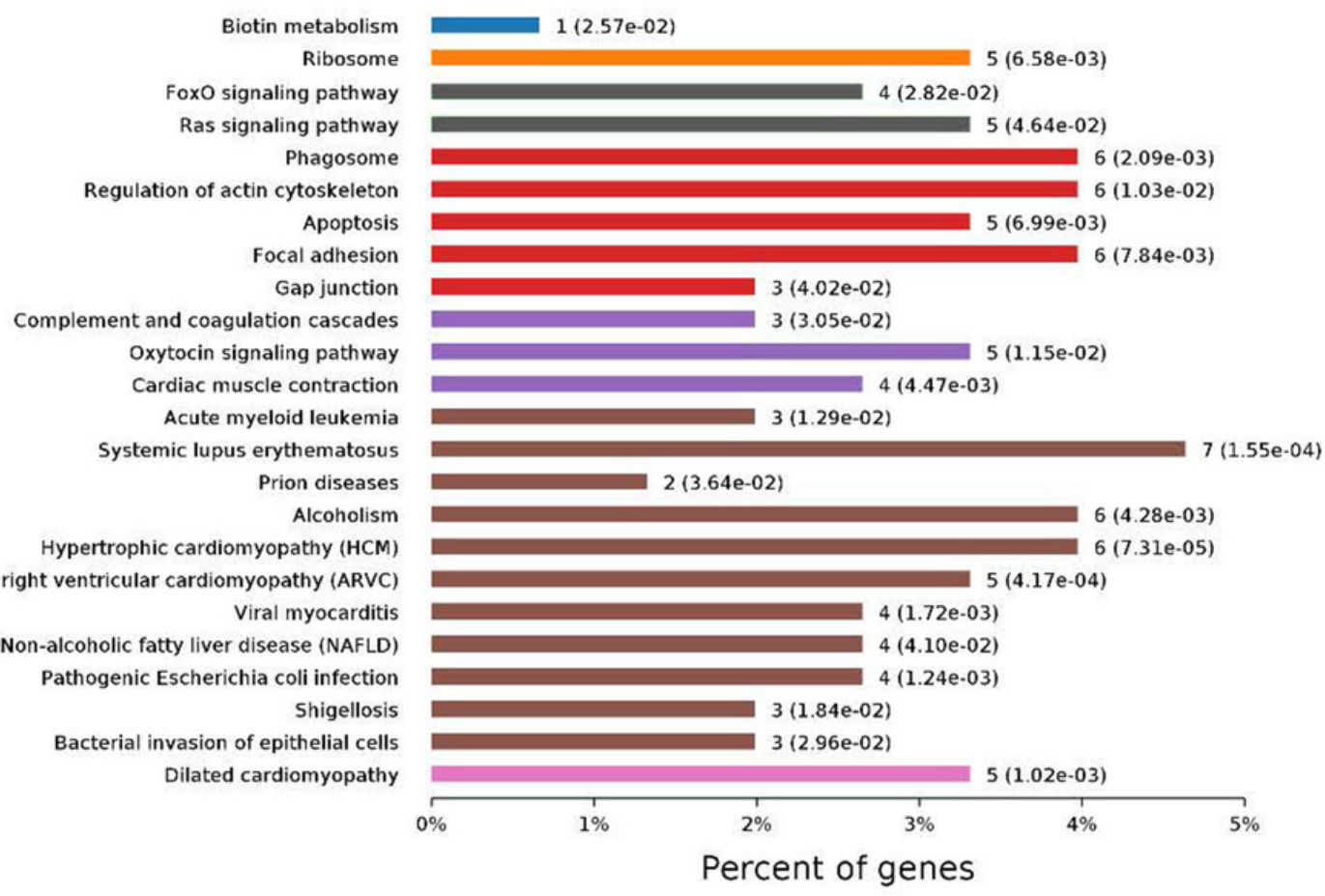

A : Metabolism

B : Genetic Information Processing

C : Environmental Information Processing

D : Cellular Processes

E : Organismal Systems

F : Human Diseases
$\mathrm{AH}$ : Metabolism of cofactors and vitamins

BB : Translation

CB : Signal transduction

DA : Transport and catabolism

DB : Cell motility

DC : Cell growth and death

DD : Cellular community

EA : Immune system

$\mathrm{EB}$ : Endocrine system

EC : Circulatory system

FB : Cancers: Specific types

$\mathrm{FC}$ : Immune diseases

FD : Neurodegenerative diseases

$\mathrm{FE}$ : Substance dependence

FF : Cardiovascular diseases

FG : Endocrine and metabolic diseases

FH : Infectious diseases: Bacterial

$\mathrm{HA}$ : Other and unknow 
Figure 6

Changes in the hypertrophic cardiomyopathy signaling pathway in lung tissue of patients with pulmonary hypertension.

Upregulated proteins are highlighted in red boxes.

HYPERTROPHIC CARDIOMYOPATHY (HCM)

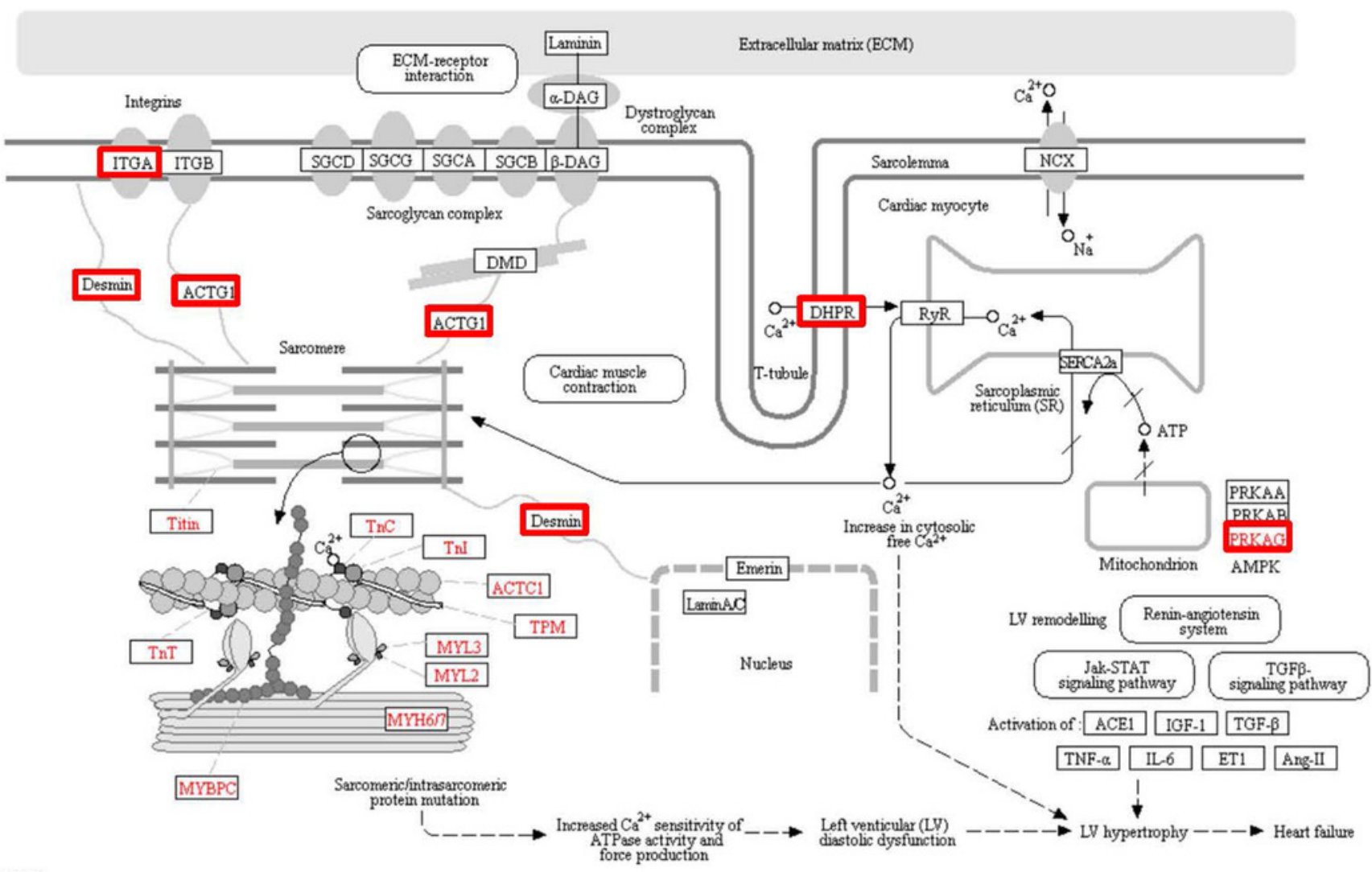


Figure 7

Changes in the arrhythmogenic right ventricular cardiomyopathy signaling pathway in lung tissue of patients with pulmonary hypertension.

Upregulated proteins are highlighted in red boxes.

ARRHYTHMOGENIC RIGHT VENTRICULAR CARDIOMYOPATHY(ARVC)

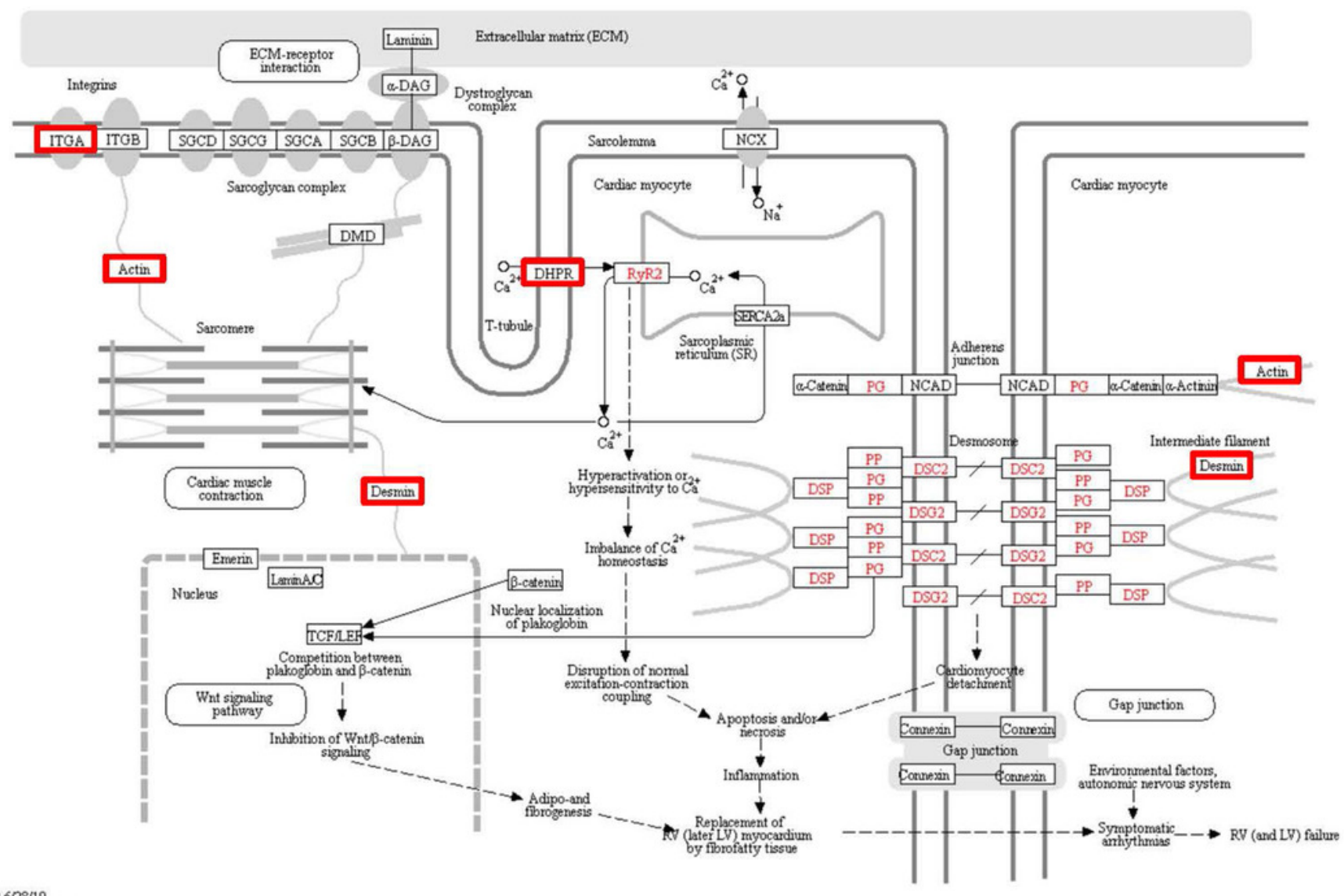


Figure 8

Changes in the viral myocarditis signaling pathway in lung tissue of patients with pulmonary hypertension.

Upregulated proteins are highlighted in red boxes.

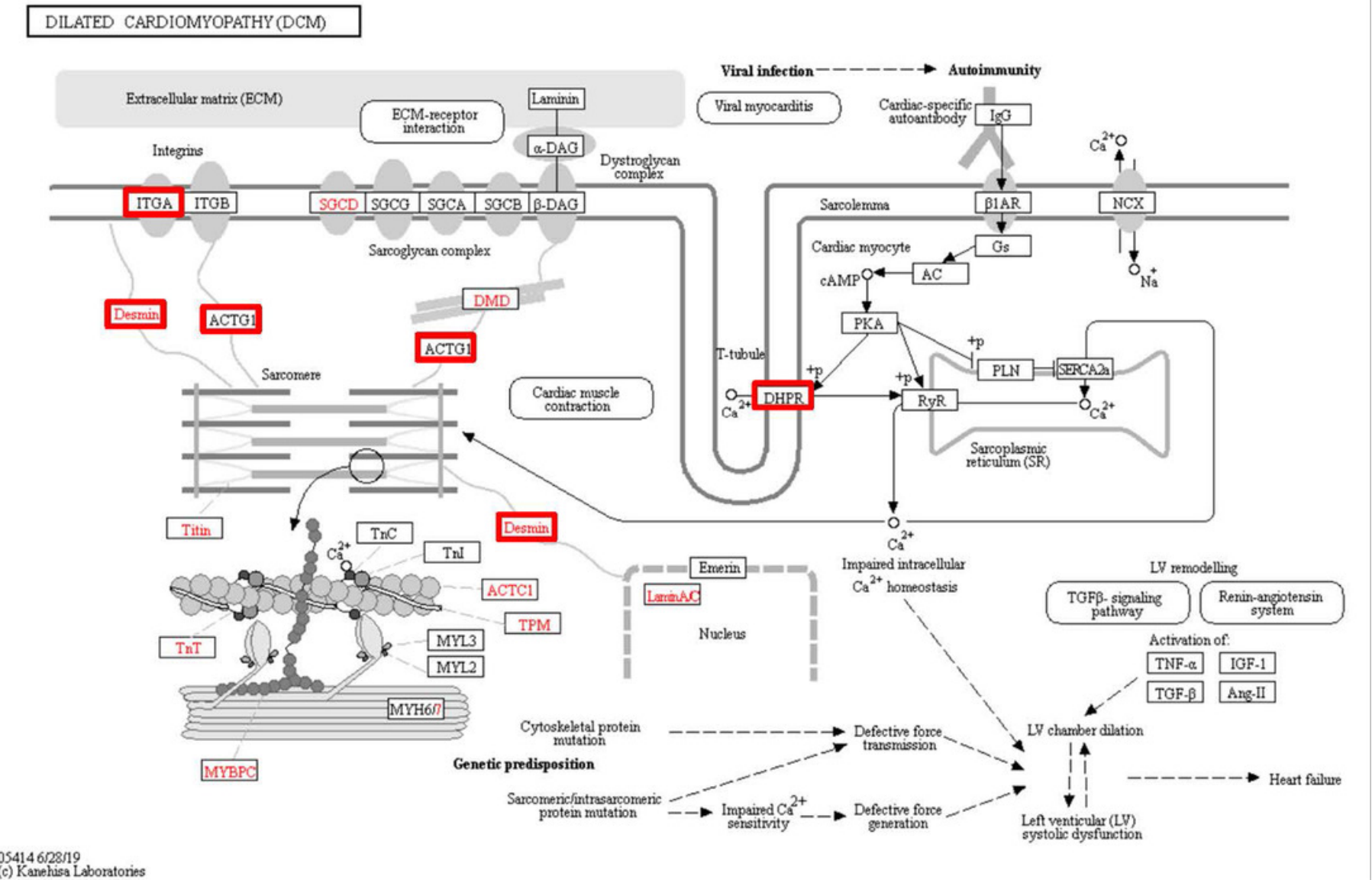


Figure 9

Protein-protein interaction networks in the Kyoto Encyclopedia of Genes and Genomes pathways that are ranked as the top 10 regulatory pathways.

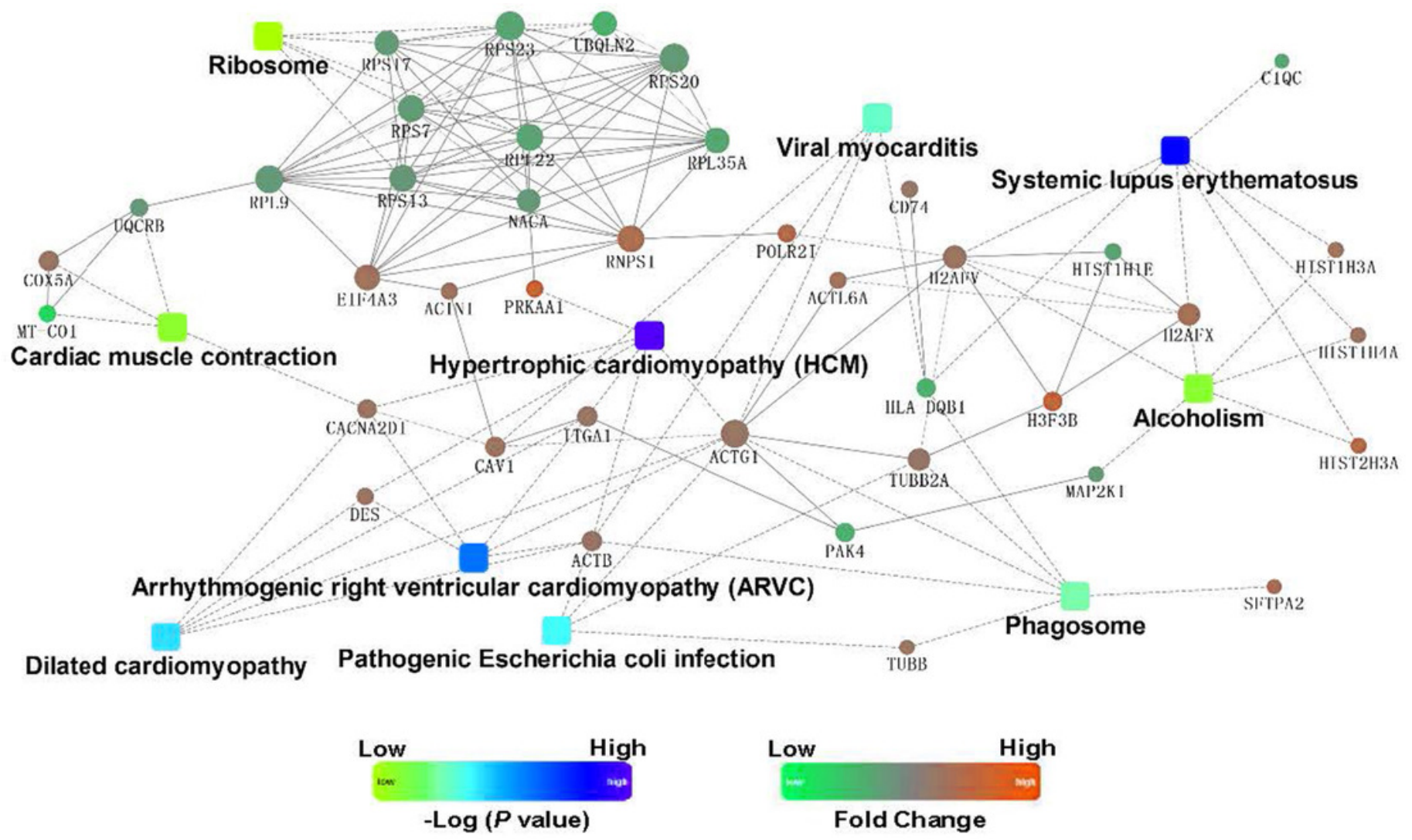




\section{Table $\mathbf{1}$ (on next page)}

Results of protein identification analyses 
1 Table 1. Results of protein identification analyses

\begin{tabular}{clllll}
\hline Unused & Proteins & Proteins & Distinct & Spectra & \% of \\
ProtScore & detected, & before & peptides, & identified, & Total \\
(Conf) cutoff & No. & grouping, No. & No. & No. & spectra \\
\hline$>\mathbf{2 . 0}(\mathbf{9 9 )}$ & 2502 & 8613 & 35550 & 119806 & 46.2 \\
$\mathbf{> 1 . 3}(\mathbf{9 5})$ & 3118 & 12147 & 36406 & 121271 & 46.8 \\
$\mathbf{> 0 . 4 7}(\mathbf{6 6})$ & 3251 & 12682 & 36614 & 121540 & 46.9 \\
$\mathbf{C u t o f f}>\mathbf{0 . 0 5}$ & & & & & \\
$(\mathbf{1 0} \%)$ applied & & 14210 & 37010 & 122026 & 47.1 \\
\hline
\end{tabular}

2 Note: Using an Unused ProtScore cutoff $>1.3$ and peptides $\geq 1$, we obtained 2953 proteins. 
Table 2 (on next page)

Fold changes of key proteins in pulmonary hypertensive lung tissue 
1 Table 2. Fold changes of key proteins in pulmonary hypertensive lung tissue

\begin{tabular}{lll}
\hline Protein ID (UniProt) & Gene Symbol & Fold Change \\
\hline P54289 & CACNA2D1 & 1.246 \\
P17661 & DES & 1.257 \\
Q13131 & PRKAA1 & 1.794 \\
P60709 & ACTB & 1.215 \\
P56199 & ITGA1 & 1.209 \\
P63261 & ACTG1 & 1.215 \\
P52566 & ARHGDIB & 0.743 \\
\hline
\end{tabular}

2 